\title{
A RESULT ON THE BIFURCATION FROM THE PRINCIPAL EIGENVALUE OF THE $A p$-LAPLACIAN
}

\author{
P. DRÁBEK*, A. ELKHALIL AND A. TOUZANI
}

Abstract. We study the following bifurcation problem in any bounded domain $\Omega$ in $\mathbf{R}^{N}$ :

$$
\left\{\begin{array}{l}
A_{p} u:=-\sum_{\substack{i, j=1 \\
\lambda g(x)|u|^{p-2}}}^{N} \frac{\partial}{\partial x_{i}}\left[\left(\sum_{m, k=1}^{N} a_{m k}(x) \frac{\partial u}{\partial x_{m}} \frac{\partial u}{\partial x_{k}}\right)^{\frac{p-2}{2}} a_{i j}(x) \frac{\partial u}{\partial x_{j}}\right]= \\
u \in W_{0}^{1, p}(\Omega) .
\end{array}\right.
$$

We prove that the principal eigenvalue $\lambda_{1}$ of the eigenvalue problem

$$
\left\{\begin{array}{l}
A_{p} u=\lambda g(x)|u|^{p-2} u \\
u \in W_{0}^{1, p}(\Omega),
\end{array}\right.
$$

is a bifurcation point of the problem mentioned above.

\section{INTRODUCTION}

In this paper we study the bifurcation problem

$$
\left\{\begin{array}{l}
A_{p} u=\lambda g(x)|u|^{p-2} u+f(u, u, \lambda), \\
u \in W_{0}^{1, p}(\Omega)
\end{array}\right.
$$

1991 Mathematics Subject Classification. 35B32, 35J70, 35P30.

Key words and phrases. $A_{p}$-Laplacian, indefinite weight, the first eigenvalue, bifurcation problem.

Received: July 1, 1997.

* The first author was partially supported by the Grant \#201/97/0395 of the Grant Agency of the Czech Republic as well as The Ministry of Education of Czech Republic Project No. VS97156. 
where $\Omega$ is a bounded domain in $\mathbb{R}^{N}, N \geq 1 ; g \in L_{\text {loc }}^{\infty}(\Omega) \cap L^{r}(\Omega)$ is an indefinite weight function, with $r=r(N, p)$ satisfying the conditions

$$
\begin{cases}r>N p^{\prime} & \text { for } 1<p \leq N, \\ r=1 & \text { for } p>N .\end{cases}
$$

We assume that $\left|\Omega^{+}\right| \neq 0$ with $\Omega^{+}=\{x \in \Omega ; g(x)>0\}$. The so-called $A_{p}$-Laplacian is defined by

$$
\begin{aligned}
A_{p} u & =-\sum_{i, j=1}^{N} \frac{\partial}{\partial x_{i}}\left[\left(\sum_{m, k=1}^{N} a_{m k}(x) \frac{\partial u}{\partial x_{m}} \frac{\partial u}{\partial x_{k}}\right)^{\frac{p-2}{2}} a_{i j}(x) \frac{\partial u}{\partial x_{j}}\right] \\
& =-\operatorname{div}\left(|\nabla u|_{a}^{p-2} A(\nabla u)\right),
\end{aligned}
$$

where $A=\left(a_{i j}(x)\right)_{1 \leq i, j \leq N}$ is a matrix satisfying the conditions

$$
\begin{cases}a_{i j} \equiv a_{j i} \in L^{\infty}(\Omega) \cap C_{\mathrm{loc}}^{1, \gamma}(\Omega), & 0<\gamma \leq 1, \forall i, j=1, \ldots, N, \\ |\xi|_{a}^{2}:=\sum_{i, j=1}^{N} a_{i j}(x) \xi_{i} \xi_{j} \geq|\xi|^{2}, & \forall x \in \Omega, \forall \xi \in \mathbb{R}^{N} .\end{cases}
$$

Nonlinearity $f$ is a function satisfying some conditions to be specified later.

Problems involving the $A_{p}$-Laplacian, have been studied in $[\mathrm{M}, \mathrm{L}-\mathrm{T}, \mathrm{T}$, E, E-Li-T]. We note that bifurcation problem is not considered there.

Bifurcation problem of the type (1.1), with $a_{i j} \equiv \delta_{i j}, \forall i, j=1, \ldots, N$, and other conditions on $g$ and $f$, were studied on bounded domains by [B-H], $[\mathrm{D} 1, \mathrm{D} 2]$ and $[\mathrm{D}-\mathrm{M}]$. The later authors consider the regular bounded domain with $\partial \Omega$ of class $C^{2, \beta}$ for some $\left.\beta \in\right] 0,1[$ and $g \equiv 1$. This result was extended for the bounded domain having the segment property and $g \in L^{\infty}(\Omega)$ by $[\mathrm{E}$, E-La-T]. The case $\Omega=\mathbb{R}^{N}$ was studied by [D-H] (cf. also [D-K-N]) under some appropriate conditions on $f$ and $g$.

In this work we investigate the situation improving the conditions on $f$ and $g$ for any bounded domain. This paper is organized as follows: in Section 2, we introduce some assumptions and notations which we use later and prove some technical preliminaries. In Section 3, we verify that the topological degree is well defined for our operators. We also show that the topological degree has a jump when $\lambda$ crosses $\lambda_{1}$, which implies the bifurcation result.

\section{Assumptions, Definitions and Preliminaries}

We first introduce some basic definitions, assumptions and notations. For every $x$ fixed in $\Omega$ denote

$$
\langle\xi, \eta\rangle_{a}=\sum_{i, j=1}^{N} a_{i j}(x) \xi_{i} \eta_{j}, \forall \xi, \eta \in \mathbb{R}^{N} .
$$

The symbol $|\cdot|_{a}$ denotes the norm induced by $\langle\cdot, \cdot\rangle_{a}$. We use $W_{0}^{1, p}(\Omega)$-norm defined by

$$
\|v\|_{1, p}=\left\||\nabla v|_{a}\right\|_{p}=\left(\int_{\Omega}|\nabla v(x)|_{a}^{p} d x\right)^{\frac{1}{p}} .
$$


Denote for $t \in] 1,+\infty\left[, t^{\prime}=\frac{t}{t-1} ; t^{*}=\frac{N t}{N-t}\right.$ if $1<t<N$ and $t^{*}=\infty$ if $N \leq t<\infty$.

2.1. Assumptions. We assume that

$\left(f_{1}\right) \quad f: \Omega \times \mathbb{R} \times \mathbb{R} \rightarrow \mathbb{R}$ satisfies Caratheodory's conditions in the first two variables and

$$
f(x, s, \lambda)=o\left(|s|^{p-1}\right) \text { for } s \rightarrow 0
$$

uniformly a.e. with respect to $x$ and uniformly with respect to $\lambda$ in bounded sets of $\mathbb{R}$;

$\left(f_{2}\right) \quad$ there is a $\left.q \in\right] p, p^{*}[$ such that

$$
\lim _{|s| \rightarrow+\infty} \frac{|f(x, s, \lambda)|}{|s|^{q-1}}=0,
$$

uniformly a.e. with respect to $x$ and uniformly with respect to $\lambda$ in bounded sets.

2.2. Definitions. 1. By a solution of (1.1) we understand a pair $(\lambda, u)$ in $\mathbb{R} \times W_{0}^{1, p}(\Omega)$ satisfying (1.1) in the weak sense, i.e., such that

$$
\int_{\Omega}|\nabla u|_{a}^{p-2}\langle\nabla u, \nabla v\rangle_{a} d x=\int_{\Omega}\left[\lambda g(x)|u|^{p-2} u+f(x, u, \lambda)\right] v d x
$$

for all $v \in W_{0}^{1, p}(\Omega)$. We note that the pair $(\lambda, 0)$ is a solution of (1.1) for every $\lambda \in \mathbb{R}$. The pairs of this form will be called the trivial solutions of (1.1). We say that $P=(\bar{\lambda}, 0)$ is a bifurcation point of (1.1) if in any neighborhood of $P$ in $\mathbb{R} \times W_{0}^{1, p}(\Omega)$ there exists a nontrivial solution of (1.1).

2. Let $X$ be a real reflexive Banach space and let $X^{*}$ stand for its dual with respect to the pairing $\langle\cdot, \cdot\rangle$. We shall deal with mappings $T$ acting form $X$ into $X^{*}$. The strong convergence in $X$ (and in $X^{*}$ ) is denoted by $\rightarrow$ and the weak convergence by $\rightarrow$, respectively. $T$ is demicontinuous at $u$ in $X$, if $u_{n} \rightarrow u$ in $X$, implies that $T u_{n} \rightarrow T u$ in $X^{*} . T$ is said to belong to the class $\left(S_{+}\right)$, if for any sequence $\left\{u_{n}\right\}$ in $X$ with $u_{n} \rightarrow u$ and $\limsup _{n \rightarrow+\infty}\left\langle T u_{n}, u_{n}-u\right\rangle \leq 0$, it follows that $u_{n} \rightarrow u$ in $X$. We write $T \in\left(S_{+}\right)$.

2.3. Degree theory. If $T \in\left(S_{+}\right)$and $T$ is demicontinuous, then it is possible to define the degree $\operatorname{Deg}[T ; D, 0]$, where $D \subset X$ is a bounded open set such that $T u \neq 0$ for any $u \in \partial D$. Its properties are analogous to the ones of the Leray-Schauder degree (cf. [B], [S] or [B-P]).

A point $u_{0} \in X$ will be called a critical point of $T$ if $T u_{0}=0$. We say that $u_{0}$ is an isolated critical point of $T$ if there exists $\varepsilon>0$ such that for any $u \in B_{\varepsilon}\left(u_{0}\right), T u \neq 0$ if $u \neq u_{0}$. Then the limit

$$
\text { Ind }\left(T, u_{0}\right)=\lim _{\varepsilon \rightarrow 0^{+}} \operatorname{Deg}\left[T ; B_{\varepsilon}\left(u_{0}\right), 0\right]
$$

exists and is called the index of the isolated critical point $u_{0}$. 
Assume, furthermore, that $T$ is a potential operator, i.e. for some continuously differentiable functional $\Phi: X \rightarrow \mathbb{R}, \Phi^{\prime}(u)=T u, u \in X$. Then we have the following two lemmas which we can find in [D1], [D2] or [D-H].

Lemma 2.1. Let $u_{0}$ be a local minimum of $\Phi$ and an isolated critical point of $T$. Then

$$
\text { Ind }\left(T, u_{0}\right)=1 \text {. }
$$

Lemma 2.2. Assume that $\langle T u, u\rangle>0$ for all $u \in X,\|u\|_{X}=\rho$. Then

$$
\operatorname{Deg}\left[T ; B_{\rho}(0), 0\right]=1 .
$$

2.4. Preliminaries. Define operators $A_{p}, G: W_{0}^{1, p}(\Omega) \rightarrow W^{-1, p^{\prime}}(\Omega)$ and $F: \mathbb{R} \times W_{0}^{1, p}(\Omega) \rightarrow W_{0}^{-1, p^{\prime}}(\Omega)$, by

$$
\begin{aligned}
\left\langle A_{p} u, v\right\rangle & =\int_{\Omega}|\nabla u(x)|_{a}^{p-2}\langle\nabla u(x), \nabla v(x)\rangle_{a} d x \\
\langle G u, v\rangle & =\int_{\Omega} g(x)|u(x)|^{p-2} u(x) v(x) d x \\
\langle F(\lambda, u), v\rangle & =\int_{\Omega} f(x, u(x), \lambda) v(x) d x
\end{aligned}
$$

for any $u, v \in W_{0}^{1, p}(\Omega)$.

Remark 2.3. (i) Due to (2.3) the function $u$ is a weak solution of (1.1) if and only if

$$
A_{p} u-\lambda G u-F(\lambda, u)=0 \quad \text { in } W^{-1, p^{\prime}}(\Omega) .
$$

(ii) The operator $A_{p}$ has the following properties: $A_{p}$ is odd, $(p-1)$-homogeneous, strictly monotone, i.e.,

$$
\left\langle A_{p} u-A_{p} v, u-v\right\rangle>0 \text { for all } u \neq v,
$$

and $A_{p} \in\left(S_{+}\right)$(cf. $\left.[T]\right)$. We have also

$$
\left\|A_{p} u\right\|_{W^{-1, p^{\prime}(\Omega)}}=\left\||\nabla u|_{a}\right\|_{p}^{p-1}
$$

for any $u \in W_{0}^{1, p}(\Omega)$.

Lemma 2.4. $G$ is compact, odd and $(p-1)$-homogeneous.

Proof. Step 1 Definition of $G$.

First case: if $1<p<N, r>N p^{\prime}$. Let $u, v \in W_{0}^{1, p}(\Omega)$. By Hölder's inequality, we have

$$
\left.\left|\int_{\Omega} g(x)\right| u(x)\right|^{p-2} u(x) v(x) d x \mid \leq\|g\|_{r}\|u\|_{s}^{p-1}\|v\|_{p^{*}},
$$

where $s$ is given by

$$
\frac{p-1}{s}+\frac{1}{p^{*}}+\frac{1}{r}=1
$$


Therefore

$$
\frac{p-1}{s}=1-\frac{1}{r}-\frac{1}{p^{*}}>1-\frac{1}{N p^{\prime}}-\frac{1}{p^{*}}>1-\frac{p}{N}-\frac{1}{p^{*}}=\frac{p-1}{p^{*}} .
$$

i.e.

$$
p-1<s<p^{*}
$$

Then it suffices that

$$
\max (1, p-1)<s<p^{*}
$$

and $G$ is well defined.

Second case: if $p=N, r>N N^{\prime}=N+N^{\prime}$. In this case

$$
W_{0}^{1, N}(\Omega) \hookrightarrow L^{\bar{q}}(\Omega),
$$

for any $\bar{q} \in\left[1,+\infty\left[\right.\right.$. Since $r>N$, there is $\bar{q}>1$ such that $\frac{1}{\bar{q}}+\frac{1}{r}+\frac{1}{N^{\prime}}=1$.

We obtain that

$$
\bar{q}=\frac{1}{1-\left(\frac{r+N^{\prime}}{r N^{\prime}}\right)} .
$$

By Hölder's inequality, we arrive at

$$
\left.\left|\int_{\Omega} g(x)\right| u(x)\right|^{N-2} u(x) v(x) d x \mid \leq\|g\|_{r}\|u\|_{N}^{N-1}\|v\|_{\bar{q}},
$$

for any $u, v$ in $W_{0}^{1, N}(\Omega)$. Then in this case $G$ is well defined.

Third case: if $p>N, r=1$. In this case

$$
W_{0}^{1, p}(\Omega) \hookrightarrow C(\bar{\Omega}) \cap L^{\infty}(\Omega) .
$$

Then for any $u, v \in W_{0}^{1, p}(\Omega)$, we have

$$
\left.\left|\int_{\Omega} g(x)\right| u(x)\right|^{p-2} u(x) v(x) d x \mid<\infty,
$$

with $g \in L^{1}(\Omega)$, and $G$ is well defined also in this case.

Step 2 Compactness of $G$. Let $\left(u_{n}\right) \subset W_{0}^{1, p}(\Omega)$ be a sequence such that $u_{n} \rightarrow u$ weakly in $W_{0}^{1, p}(\Omega)$. We must show that $G u_{n} \rightarrow G u$ strongly in $W_{0}^{1, p}(\Omega)$, i.e.

$$
\sup _{\substack{v \in W_{0}^{1, p}(\Omega) \\\left\||\nabla v|_{a}\right\|_{p} \leq 1}}\left|\int_{\Omega} g\left[\left|u_{n}\right|^{p-2} u_{n}-|u|^{p-2} u\right] v d x\right|=0(1), \quad n \rightarrow+\infty .
$$

If $1<p<N, r>N p^{\prime}$ : Let $s$ be as in (2.7). Then

$$
\begin{aligned}
& \sup _{\substack{v \in W_{0}^{1, p}(\Omega) \\
\left\||\nabla v|_{a}\right\|_{p} \leq 1}}\left|\int_{\Omega} g\left[\left|u_{n}\right|^{p-2} u_{n}-|u|^{p-2} u\right] v d x\right| \\
& \leq \sup _{\substack{v \in W_{0}^{1, p}(\Omega) \\
\left\||\nabla v|_{a}\right\|_{p} \leq 1}}\left[\|g\|_{r}\left\|\left|u_{n}\right|^{p-2} u_{n}-|u|^{p-2} u\right\|_{\frac{s}{p-1}}\|v\|_{p^{*}}\right] \\
& \leq c\|g\|_{r}\left\|\left|u_{n}\right|^{p-2} u_{n}-|u|^{p-2} u\right\|_{\frac{s}{p-1}}
\end{aligned}
$$


where $c$ is the constant of Sobolev's embedding. We have

$$
\left\|\left|u_{n}\right|^{p-2} u_{n}-|u|^{p-2} u\right\|_{\frac{s}{p-1}}=o(1) \text {, as } n \rightarrow+\infty
$$

due to the continuity of Nemytskii's operator $u \mapsto|u|^{p-2} u$ from $L^{s}(\Omega)$ into $L^{\frac{s}{p-1}}(\Omega)$. Rellich's theorem yields that $u_{n} \rightarrow u$ weakly in $W_{0}^{1, p}(\Omega)$ implies that $u_{n} \rightarrow u$ strongly in $L^{s}(\Omega)$ because $\max (1, p-1)<s<p^{*}$. The compactness of $G$ then follows.

$$
\begin{aligned}
& \text { If } p=N, r>N+N^{\prime}=N N^{\prime}: \\
& \left|\int_{\Omega} g\left[\left|u_{n}\right|^{N-2} u_{n}-|u|^{N} u\right] v d x\right| \leq\|g\|_{r}\left\|\left|u_{n}\right|^{N-2} u_{n}-|u|^{N-2} u\right\|_{N}^{N-1}\|v\|_{\bar{q}},
\end{aligned}
$$

where $\bar{q}$ is given by (2.8). By Sobolev's embedding, there is $c>0$ such that

$$
\|v\|_{\bar{q}} \leq c\left\||\nabla v|_{a}\right\|_{N}, \quad \forall v \in W_{0}^{1, N}(\Omega) .
$$

Thus

$$
\begin{aligned}
& \sup _{\substack{\||\nabla v| a\|_{N} \leq 1 \\
v \in W_{0}^{1, p}(\Omega)}}\left|\int_{\Omega} g\left[\left|u_{n}\right|^{N-2} u_{n}-|u|^{N-2} u\right] v d x\right| \\
& \leq C\|g\|_{r}\left\|\left|u_{n}\right|^{N-2} u_{n}-|u|^{N-2} u\right\|_{N}^{N-1} .
\end{aligned}
$$

From the continuity of $u \mapsto|u|^{N-2} u$ from $L^{N}(\Omega)$ into $L^{N^{\prime}}(\Omega)$, and from the compact embedding of $W_{0}^{1, N}(\Omega)$ in $L^{N}(\Omega)$, we have the desired result.

If $p>N, r=1$. By Rellich's embedding theorem of $W_{0}^{1, p}(\Omega)$ into $C(\bar{\Omega})$, we obtain

$$
\begin{aligned}
& \sup _{\substack{\|\mid \nabla v\|_{a} \|_{p} \leq 1 \\
v \in W_{0}^{1, p(\Omega)}}}\left|\int_{\Omega} g\left[\left|u_{n}\right|^{p-2} u_{n}-|u|^{p-2} u\right] v d x\right| \\
& \leq C\|g\|_{1} \sup _{\bar{\Omega}}\left|\| u_{n}\right|^{p-2} u_{n}-|u|^{p-2} u \mid,
\end{aligned}
$$

where $C$ is the constant given by embedding of $W_{0}^{1, p}(\Omega)$ in $C(\bar{\Omega}) \cap L^{\infty}(\Omega)$. It is clear that

$$
\left.\sup _{\bar{\Omega}}|| u_{n}\right|^{p-2} u_{n}-|u|^{p-2} u \mid=o(1) \text {, as } n \rightarrow+\infty \text {. }
$$

The oddness and ( $p-1)$-homogeneity of $G$ is obvious. Thus the lemma is proved.

Lemma 2.5. $F(\lambda, \cdot)$ is compact, $F(\lambda, 0)=0$ and we have

$$
\lim _{\left\||\nabla u|_{a}\right\|_{p} \rightarrow 0} \frac{F(\lambda, u)}{\left\||\nabla u|_{a}\right\|_{p}^{p-1}}=0 \quad \text { in } W^{-1, p^{\prime}}(\Omega),
$$

uniformly for $\lambda$ is in a bounded subset of $\mathbb{R}$.

Proof. (2.1) and (2.2) imply that for any $\varepsilon>0$, there are two reals $\delta=\delta(\varepsilon)$ and $M=M(\delta)$ such that for a.e. $x \in \Omega$, we have

$$
|f(x, s, \lambda)| \leq \varepsilon|s|^{p-1} \quad \text { for }|s| \leq \delta
$$


and

$$
|f(x, s, \lambda)| \leq M|s|^{q-1} \text { for }|s| \geq \delta .
$$

Therefore, for $0<\varepsilon \leq 1$, we obtain

$$
\begin{aligned}
& \int_{\Omega}|f(x, u(x), \lambda)|^{q^{\prime}} d x \\
& =\int_{\{x,|u(x)| \leq \delta\}}|f(x, u(x), \lambda)|^{q^{\prime}} d x+\int_{\{x,|u(x)| \geq \delta\}}|f(x, u(x), \lambda)|^{q^{\prime}} d x \\
& \leq \int_{\Omega}|u(x)|^{q^{\prime}(p-1)} d x+M \int_{\Omega}|u(x)|^{q} d x .
\end{aligned}
$$

We have $q^{\prime}(p-1) \leq p^{\prime}(p-1)=p<q$. So $L^{q}(\Omega) \hookrightarrow L^{q^{\prime}(p-1)}(\Omega)$ and there is $c>0$ such that

$$
\int_{\Omega}|u(x)|^{q^{\prime}(p-1)} d x \leq c \int_{\Omega}|u(x)|^{q} d x .
$$

We deduce that

$$
\int_{\Omega}|f(x, u(x), \lambda)|^{q^{\prime}} d x \leq(c+M) \int_{\Omega}|u(x)|^{q} d x .
$$

Hence $u \mapsto F(\lambda, u)$ maps $L^{q}(\Omega)$ into $L^{q^{\prime}}(\Omega)$. Moreover, if $u_{n} \rightarrow u$ in $W_{0}^{1, p}(\Omega)$, $u_{n} \rightarrow u$ in $L^{q}(\Omega)$ (because $p<q<p^{*}$ ) and $F\left(\lambda, u_{n}\right) \rightarrow F(\lambda, u)$ in $L^{q^{\prime}}(\Omega)$. Since $L^{q^{\prime}}(\Omega) \hookrightarrow W^{-1, p^{\prime}}(\Omega)$, we have $F\left(\lambda, u_{n}\right) \rightarrow F(\lambda, u)$ in $W^{-1, p^{\prime}}(\Omega)$. This proves that $F(\lambda, \cdot)$ is compact. It is clear that $F(\lambda, 0)=0$ for any $\lambda \in \mathbb{R}$.

By $\left(f_{2}\right)$, we have $\frac{F(\lambda, u)}{\left\||\nabla u|_{a}\right\|_{p}^{p-1}} \rightarrow 0$ in $L^{q^{\prime}}(\Omega)$. Indeed, set $v=\frac{u}{\left\||\nabla u|_{a}\right\|_{p}}$. Then

$$
\frac{F(\lambda, u)}{\left\||\nabla u|_{a}\right\|_{p}^{p-1}}=\frac{F(\lambda, u)}{|u|^{p-1}}|v|^{p-1} .
$$

From (2.12) and Hölder's inequality, we deduce that

$$
\int_{\Omega}\left|\frac{F(\lambda, u)}{\left\||\nabla u|_{a}\right\|_{p}^{p-1}}\right|^{q^{\prime}} d x \leq\left(\int_{\Omega}\left|\frac{F(\lambda, u)}{|u|^{p-1}}\right|^{q^{\prime} t} d x\right)^{\frac{1}{t}}\left(\int_{\Omega}|v|^{(p-1) q^{\prime} t^{\prime}} d x\right)^{\frac{1}{t^{\prime}}}
$$

for some $t>0$ which satisfies

$$
\frac{q^{\prime}(q-p)}{p^{*}}<\frac{1}{t}<\frac{p^{*}-(p-1) q^{\prime}}{p^{*}} .
$$

This is always possible, since $p<q<p^{*}$. By (2.10) and (2.11), we obtain that

$$
\left\|\left|\frac{F(\lambda, u)}{|u|^{p-1}}\right|^{q^{\prime}}\right\|_{t}^{t} \leq \varepsilon|\Omega|+M^{q^{\prime} t} \int_{\Omega}|u|^{q^{\prime} t(q-p)} d x, \quad \forall \varepsilon>0 .
$$

From this inequality and since $u \rightarrow 0$ in $W_{0}^{1, p}(\Omega)$, we have by $(2.13)$ that

$$
\left\|\left|\frac{F(\lambda, u)}{|u|^{p-1}}\right|^{q^{\prime}}\right\|_{t}^{t} \rightarrow 0, \text { as } u \rightarrow 0 \quad \text { in } W_{0}^{1, p}(\Omega) .
$$


On the other hand, $v$ belongs to $L^{p^{*}}(\Omega)$ (because $\left\||\nabla v|_{a}\right\|_{p}=1$ ). Then we find a constant $c>0$ so that

$$
\left\||v|^{(p-1) q^{\prime}}\right\|_{t^{\prime}} \leq c
$$

since $q^{\prime} t^{\prime}(p-1)<p^{*}$ by (2.13). This concludes the proof.

Remark 2.6. Note that every continuous map $T: X \rightarrow X^{*}$ is also demicontinuous. Note also, that if $T \in\left(S_{+}\right)$then $(T+K) \in\left(S_{+}\right)$for any compact operator $K: X \rightarrow X^{*}$.

Remark 2.7. $\lambda$ is an eigenvalue of

$$
\left\{\begin{array}{l}
A_{p} u=\lambda g(x)|u|^{p-2} u \\
u \in W_{0}^{1, p}(\Omega)
\end{array}\right.
$$

if and only if the equation

$$
A_{p} u-\lambda G u=0
$$

has a solution $u \in W_{0}^{1, p}(\Omega) \backslash\{0\}$.

Now we take $T_{\lambda}=A_{p}-\lambda G-F(\lambda, \cdot)$. By Lemma 2.4, Lemma 2.5, Remark 2.3 and Remark 2.6, the degree

$$
\operatorname{Deg}\left[T_{\lambda} ; D, 0\right]
$$

(where $D$ is a bounded open set in $W_{0}^{1, p}(\Omega)$ such that $T_{\lambda} u \neq 0$ for any $u \in \partial D)$ is well defined for any $\lambda>0$.

By the same argument as used in proof of Lemma 2.4, we can show the following proposition.

Proposition 2.8. If $(\bar{\lambda}, 0)$ is a bifurcation point of problem (1.1), then $\bar{\lambda}$ is an eigenvalue of $(P)$.

\section{Bifurcation From $\lambda_{1}$}

We recall that $\lambda_{1}$ can be characterized variationally as follows:

$$
\lambda_{1}=\min \left\{\frac{\int_{\Omega}|\nabla u|_{a}^{p} d x}{\int_{\Omega} g|u|^{p} d x} ; u \in W_{0}^{1, p}(\Omega), \quad \int_{\Omega} g|u|^{p} d x>0\right\} .
$$

Recall for our problem $(P)$, (cf., $[\mathrm{L}-\mathrm{T}]$ ), that $\lambda_{1}$ is the principal eigenvalue and it is simple and isolated.

Let $E=\mathbb{R} \times W_{0}^{1, p}(\Omega)$ be equipped with the norm

$$
\|(\lambda, u)\|=\left(|\lambda|^{2}+\left\||\nabla u|_{a}\right\|_{p}^{2}\right)^{\frac{1}{2}}, \quad(\lambda, u) \in \mathbb{R} \times W_{0}^{1, p}(\Omega) .
$$

Definition 3.1. We say that

$$
C=\{(\lambda, u) \in E:(\lambda, u) \text { solves (1.1), } u \neq 0\}
$$

is a continuum of nontrivial solutions of (1.1), if it is a connected set in E. 
Theorem 3.2. Under the assumptions (1.2), (1.3), $\left(f_{1}\right)$ and $\left(f_{2}\right)$, the pair $\left(\lambda_{1}, 0\right)$ is a bifurcation point of (1.1). Moreover, there is a continuum of nontrivial solutions $C$ of (1.1) such that $\left(\lambda_{1}, 0\right) \in \bar{C}$ and $C$ is either unbounded in $E$ or there is $\bar{\lambda} \neq \lambda_{1}$, an eigenvalue of $(P)$, with $(\bar{\lambda}, 0) \in \bar{C}$.

Proof. We will give only sketch of the proof since it follows the lines of the proof of Theorem 14.18 in [D2] or Theorem 3.7 in [D-K-N]. The key point in the proof is the fact that the value of

$$
\operatorname{Deg}\left[A_{p}-\lambda G ; B_{\varepsilon}(0), 0\right]
$$

changes when $\lambda$ crosses $\lambda_{1}$. If this fact is proved then the result follows exactly as in the classical bifurcation result of Rabinowitz $[\mathrm{R}]$. Choose $\delta>0$ such that $\left(\lambda_{1}, \lambda_{1}+\delta\right)$ does not contain any eigenvalue of $(P)$. Then the variational characterization (3.1) of $\lambda_{1}$ and Lemma 2.2 yield

$$
\operatorname{Deg}\left[A_{p}-\lambda G ; B_{\varepsilon}(0), 0\right]=1,
$$

when $\lambda \in\left(\lambda_{1}-\delta, \lambda_{1}\right)$. To evaluate (3.2) for $\lambda \in\left(\lambda_{1}, \lambda_{1}+\delta\right)$ we use the following trick. Fix a number $K>0$ and define a function $\psi: \mathbb{R} \rightarrow \mathbb{R}$ by

$$
\psi(t)= \begin{cases}0 & \text { for } t \leq K, \\ \frac{2 \delta}{\lambda_{1}}(t-2 K) & \text { for } t \geq 3 K,\end{cases}
$$

and $\psi$ is positive and strictly convex in $(K, 3 K)$. Define a functional

$$
\bar{\Psi}_{\lambda}(u)=\frac{1}{p}\left\langle A_{p} u, u\right\rangle-\frac{\lambda}{p}\langle G u, u\rangle+\psi\left(\frac{1}{p}\left\langle A_{p} u, u\right\rangle\right) .
$$

Then $\bar{\Psi}_{\lambda}$ is continuously Fréchet differentiable and its critical point $u_{0} \in$ $W_{0}^{1, p}(\Omega)$ corresponds to a solution of the equation

$$
A_{p} u_{0}-\frac{\lambda}{1+\psi^{\prime}\left(\frac{1}{p}\left\langle A_{p} u_{0}, u_{0}\right\rangle\right)} G u_{0}=0 .
$$

However, since $\lambda \in\left(\lambda_{1}, \lambda_{1}+\delta\right)$, the only nontrivial critical points of $\bar{\Psi}_{\lambda}^{\prime}$ occur if

$$
\psi^{\prime}\left(\frac{1}{p}\left\langle A_{p} u_{0}, u_{0}\right\rangle\right)=\frac{\lambda}{\lambda_{1}}-1 .
$$

Due to the definition of $\psi$ we then have

$$
\frac{1}{p}\left\langle A_{p} u_{0}, u_{0}\right\rangle \in(K, 3 K)
$$

and due to (3.4) and the simplicity of $\lambda_{1}$, either $u_{0}=-u_{1}$ or $u_{0}=u_{1}$, where $u_{1}$ is the principal eigenfunction. So, for $\lambda \in\left(\lambda_{1}, \lambda_{1}+\delta\right)$, the derivative $\bar{\Psi}_{\lambda}^{\prime}$ has precisely three isolated critical points

$$
-u_{1}, 0, u_{1} \text {. }
$$

It is not difficult to prove that $\bar{\Psi}_{\lambda}$ is weakly lower semicontinuous and

$$
\lim _{\|u\|_{1, p} \rightarrow \infty} \bar{\Psi}_{\lambda}(u)=\infty
$$


due to the definition of $\psi$. So, $\bar{\Psi}_{\lambda}$ attains local minima at $u_{1}$ and $-u_{1}$. It follows from Lemma 2.1 that

$$
\operatorname{Ind}\left(\bar{\Psi}_{\lambda}^{\prime}, u_{1}\right)=\operatorname{Ind}\left(\bar{\Psi}_{\lambda}^{\prime},-u_{1}\right)=1 .
$$

Since also

$$
\left\langle\bar{\Psi}_{\lambda}^{\prime}(u), u\right\rangle>0
$$

for $\|u\|_{1, p}=R$, with $R>0$ sufficiently large, we have according to Lemma 2.2 that

$$
\operatorname{Deg}\left[\bar{\Psi}^{\prime} ; B_{R}(0), 0\right]=1 .
$$

Additivity property of the degree, (3.5) and (3.6) yield

$$
\operatorname{Deg}\left[A_{p}-\lambda G ; B_{\varepsilon}(0), 0\right]=-1
$$

for $\lambda \in\left(\lambda_{1}, \lambda_{1}+\delta\right)$ and $\varepsilon>0$ sufficiently small. Since (3.3) and (3.7) establish the "jump" of the degree the proof is complete.

\section{REFERENCES}

[B] F. E. Browder, Fixed point theory and nonlinear problems, Bull. Amer. Math. Soc. 9 (1983), 1-39.

[B-H] P. A. Binding and Y.X. Huang, Bifurcation from eigencurves of the p-Laplacian, Differential Integral Equations, 8 (1995), 415-428.

[B-P] F. E. Browder and W. F. Petryshyn, Approximation methods and the generalized topological degree for nonlinear mappings in Banach spaces, J. Funct. Anal. 3 (1969), 217-245.

[D-M] M. A. Del Pino and R. Manásevich, Global bifurcation from the eigenvalues of the p-Laplacian, J. Differential Equations, 92 (1991), 226-251.

[D1] P. Drábek, On the global bifurcation for a class of degenerate equations, Ann. Mat. Pura Appl. 159 (1991), 1-16.

[D2] P. Drábek, Solvability and Bifurcation of Nonlinear Equations, Pitman Res. Notes Math. Ser., \#264, Longman, 1992.

[D-H] P. Drábek and Y. X. Huang, Bifurcation problems for the $p$-Laplacian in $\mathbb{R}^{N}$, Trans. Amer. Math. Soc. 349 (1997), 171-188.

[D-K-N] P. Drábek, A. Kufner and F. Nicolosi: Quasilinear Elliptic Equations With Degenerations and Singularities, De Gruyter Series in Nonlinear Analysis and Applications, 5, 1997.

[E] A. Elkhalil: Sur Le probléme Non-linéaire $A_{p}$-Laplacien: Stabilité-Bifurcation, Faculté des Sciences Dhar-Mahraz, Thése De $3^{\text {eme }}$ Cycle, 1996, pp. 105.

[E-La-T] A. Elkhalil, E. Lami-Dozo and A. Touzani, Bifurcation from the first eigenvalue of the $A_{p}$-Laplacian with indefinite weight, Preprint.

[E-Li-T] A. Elkhalil, P. Lindqvist and A. Touzani, On the stability of the first eigenvalue of the problem: $-A_{p} u=\lambda g(x)|u|^{p-2} u$, with varying $p$, Preprint.

[L-T] E. Lami-Dozo and A. Touzani, Autovalores con peso indefinito del $A_{p}$ Laplaciano, Centro Latinoamericano de Matematica e Informatica (CLAMI), (1992).

[M] J. Mossino, Inégalités Isopémetriques et Applications en Physique, Hermann, Paris, 1984, pp. 182.

[R] P. H. Rabinowitz, Some global results for nonlinear eigenvalue problems, J. Funct. Anal. 7 (1971), 487-513. 
[S] I. V. Skrypnik, Methods for Analysis of Nonlinear Elliptic Boundary Value problems, Transl. Math. Monogr., AMS, \#139, 1994.

[T] A. Touzani: Quelques Résultats Sur Le Ap-Laplacien Avec Poids Indéfini, Université Libre de Bruxelles, Thése, 1992.

\section{P. DRÁBEK}

Department of Mathematics

UNIVERSITY OF WEST BOHEMIA

P.O. Box 314, 30614 PILSEN

CZECH REPUBLIC

E-mail: pdrabek@kma.zcu.cz
A. Elkhalil and A. Touzani
DÉPARTEMENT DES MATHÉmatiques
FaCulté des Sciences Dhar-Mahraz
B. P. 1796, Fes-AtLas
FEs, MOROCCO 


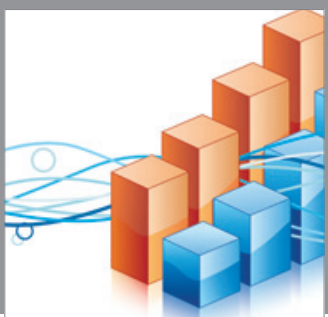

Advances in

Operations Research

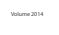

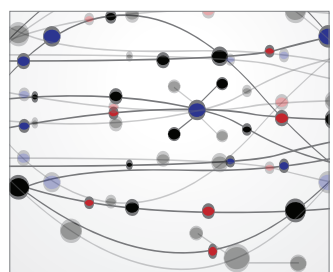

\section{The Scientific} World Journal
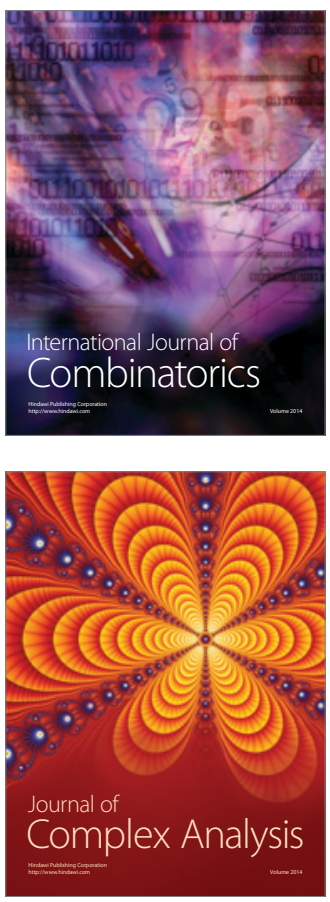

International Journal of

Mathematics and

Mathematical

Sciences
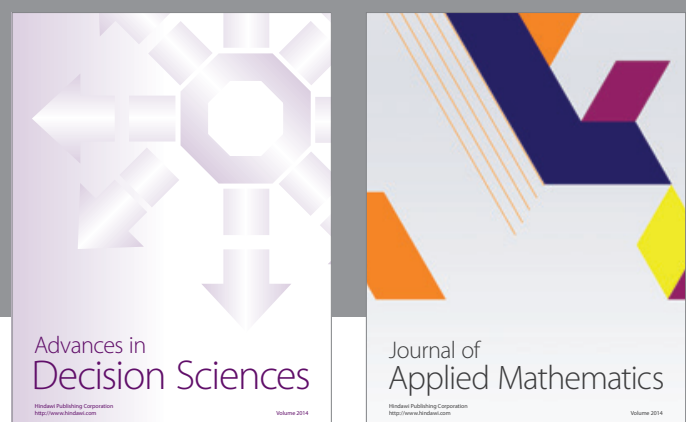

Journal of

Applied Mathematics
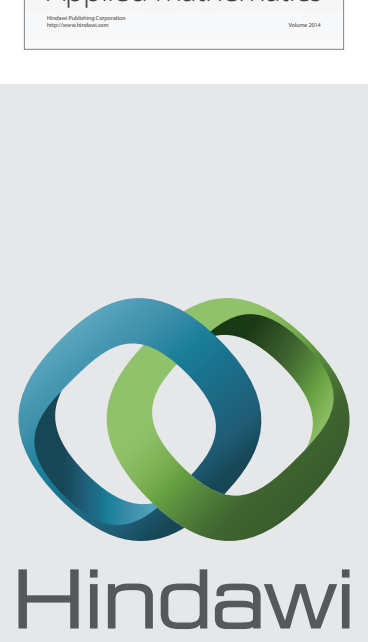

Submit your manuscripts at http://www.hindawi.com
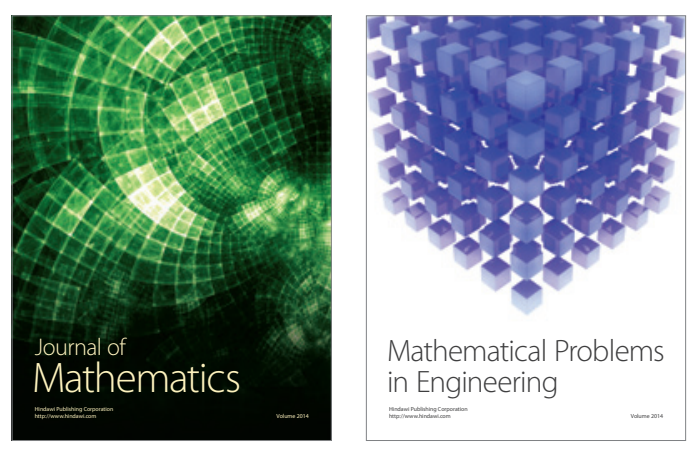

Mathematical Problems in Engineering
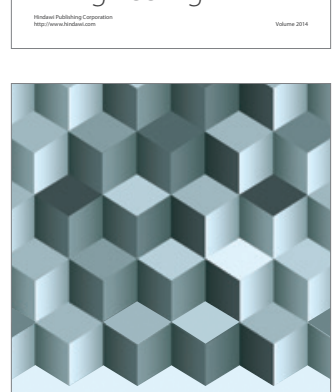

Journal of

Function Spaces
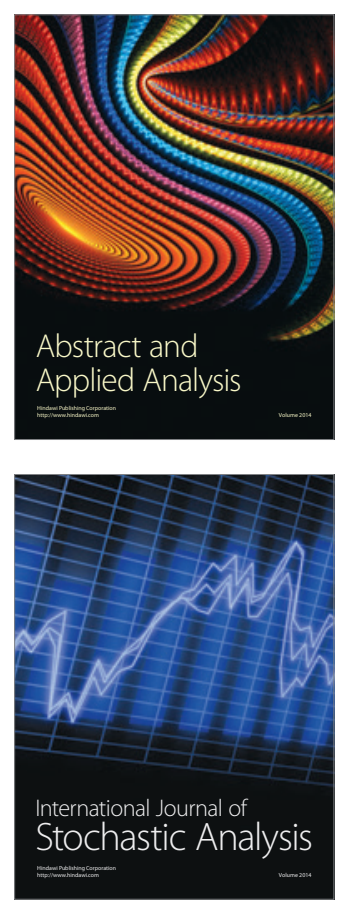

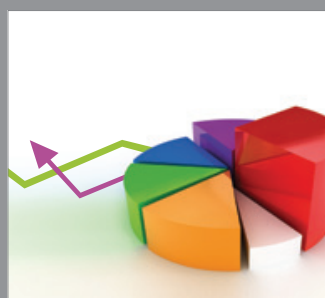

ournal of

Probability and Statistics

Promensencen
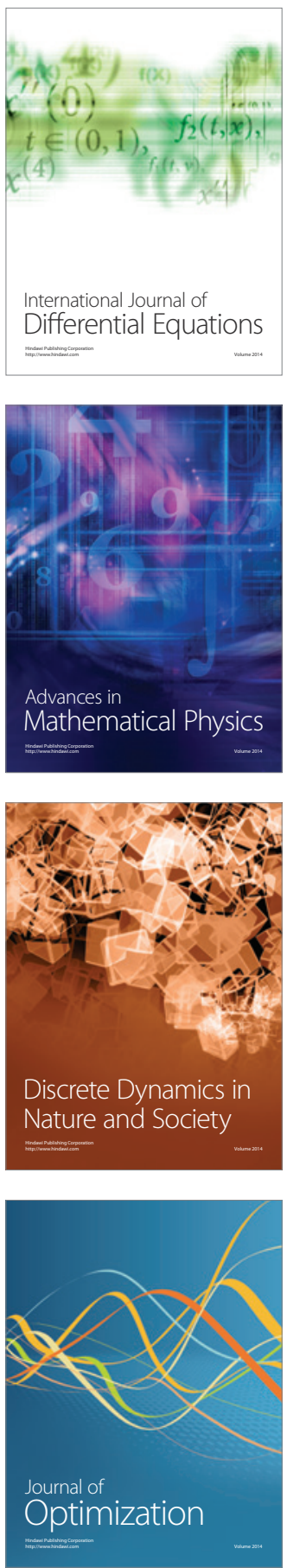